\title{
Meta-analysis of Nicorandil effectiveness on myocardial protection after percutaneous coronary intervention
}

\author{
Xiao-Tao Zhao ${ }^{1 * \dagger} \mathbb{D}$, Chun-Fei Zhang ${ }^{2 \dagger}$ and Qing-Jie Liü ${ }^{3 *}$
}

\begin{abstract}
Background: Using the current meta-analysis as well as systematic review, to determine the curative effect of Nicorandil in comparison of no Nicorandil after elective percutaneous coronary intervention(PCI) on patients.

Methods: Published literatures were identified via a computerized literature search of CENTRAL, PubMed, Cochrane, Embase Databases of Systematic Reviews. A set of randomized trials evaluating Nicorandil in comparison of no Nicorandil administered following $\mathrm{PCl}$ in patients harboring coronary artery disease were included. Outcomes were revealed based on the following parameters: peak creatine kinase-MB (CK-MB) value, left ventricular ejection fraction (LVEF), peak troponin I (cTnl), and major adverse cardiovascular events (MACEs) per randomized patients.

Results: We included a total of 14 RCTs involving 1864 subjects in the present review. According to this metaanalysis, LVEF was significantly improved in Nicorandil group; the peak CK-MB level and the incidence of adverse cardiovascular events were remarkably lower in Nicorandil group. Nicorandil and no Nicorandil administered group appeared to be equivalent with regards to cTnl.

Conclusions: Nicorandil is effective for patients undergoing elective PCI with coronary artery disease in terms of reducing the incidence of adverse cardiovascular events as well as improving heart function. Nicorandil may exert potential role as a valid and adjunctive therapy accompanied with PCl.
\end{abstract}

Keywords: Nicorandil, Percutaneous coronary intervention, Coronary artery disease, Acute myocardial infarction, Angina, Heart function, Cardiovascular events, Meta-analysis

\section{Background}

Coronary artery disease, widely acknowledged as one of the most common causes of heart disease, represent a serious health burden under the background of healthcare and socio-economic policy. Disability, morbidity, and mortality caused by CHD have been increasing year by year, with a prevalence of $10 \%$ of disability and $30 \%$ of all-cause mortality [1]. Percutaneous coronary intervention (PCI) has been known as one of the most commonly used techniques in treating coronary artery

\footnotetext{
* Correspondence: xiaotaozhao926@sina.com; qingjieliu@yahoo.com Xiao-Tao Zhao and Chun-Fei Zhang contributed equally to this paper and should be treated as co-first authors.

${ }^{1}$ Department of Cardiology, Beijing Chao-Yang Hospital, Captial Medical University, 8 Gongti Nanlu Road, Chaoyang District, Beijing 100020, NO, China ${ }^{3}$ National Institute for Radiological Protection, Chinese Center for Disease Control and Prevention, Chinese Center for Medical Response to Radiation Emergency, No. 2 Xinkang Street, Deshengmenwai, Beijing 100088, China Full list of author information is available at the end of the article
}

disease. In 1964, Dotter et al. firstly proposed the concept of percutaneous transluminal coronary angioplasty (PTCA); and the world's first case of PTCA treatment of coronary artery disease conducted by Gruntzig et al. [2] in 1977 has led to significant progress of the technology and has become the most widely used intervention therapy in treating coronary artery disease. However, potential shortcoming such as myocardial injury caused by PCI has become a major concern. Myocardial injury following PCI may attribute to distal microvascular embolization, vascular endothelial injury, coronary artery spasm, surgical procedures that block blood vessels, lateral occlusion caused by plaque displacement, reperfusion injury and tissue damage [3-5]. Several drugs were tested for the prevention or alleviation of such injuries, but beneficial outcomes have not been gained [6-8]. 
Nicorandil is a non-selective adenosine-sensitive potassium channel opener that allows the vascular smooth muscle to relax, effectively dilate the microvascular and to improve myocardial perfusion $[9,10]$. Recent basic and clinical studies revealed its protective effects for myocardial injury [11, 12]. The purpose of the current study was to provide a systematic review of Nicorandil in terms of its cardio-protective effects. The aim was to synthesize the available evidence and compare data associated with the effectiveness of Nicorandil in this objective and quantitative analysis.

\section{Methods}

\section{Literature search}

A thorough electronic literature search was performed involving randomized controlled trials (RCTs) published in databases such as Cochrane Central Register of Clinical Trials (CENTRAL), PubMed (1966-July 2017), Cochrane Database of Systematic Reviews (Issue 1, 2017), and Embase (1980-July 2017). A literature search was conducted with the use of the following keywords: 'coronary heart disease,' 'cardiovascular disease,' 'angina pectoris,' 'myocardial infarction,' 'CHD', 'Nicorandil', 'PCI', 'percutaneous coronary intervention', and 'PTCA'. In order to further maximize the literature data, we contacted the author through letters for test reports due to the lack of information or unknown details from several literature and meta-analysis. Additionally, we retrieved the bibliographies of the review articles as well as retrieved trials. The study was adhered to PRISMA guidelines.

\section{Study selection}

In order to be included in the current review, the following inclusion criteria should be met: 1) patients diagnosed with established coronary artery disease or acute myocardial infarction undergoing elective PCI; 2) comparisons of Nicorandil versus placebo or no Nicorandil administered treatment; 3) the following one or more outcome measures should be reported: peak creatine kinase-MB (CK-MB) value, Left ventricular ejection fraction (LVEF), peak troponin I (cTnI), major adverse cardiovascular events (MACEs) included i) all-cause mortality; ii) new MI; iii) any revascularization; iiii) re-hospitalization rate; 4 ) the publications were only available in English; 5) randomized controlled trials (RCTs). We considered the following studies as excluded ones: 1) studies lack of information of both PCI and Nicorandil; 2) incomplete or incorrect data, or lack of outcome; 3) observational studies or case reports; 4) repeated publications.

A review topic (or topics) was designed to include trials that were identified from the searching results of above-mentioned features. We utilized the Thomson
Research Software (EndNote X4) for the accuracy evaluation of extracted data from review. We provided original reports for further details in case of unclear information. "excluded (reason)", "pending", "Included" were indicated into the "notes" column. And authors would retrace "pending" reports from the references.

\section{Quality assessment}

We utilized Cochrane handbook for Systematic Reviews of Interventions 5.1.0, which was recommended by Cochrane Collaboration, for the assessment of study quality. The items for evaluation mainly included the following seven aspects: allocation concealment; random sequence generation; incomplete outcome data; blinding of participants and provider; selective reporting and other bias; and blinding of outcome assessment. "High risk", "low risk", and "unclear risk" were defined respectively as the evaluation of each document that was in accordance with the above seven items.

\section{Data extraction}

The first authors, the years of publication were extracted as general information. Parameters, such as object characteristics, follow-up duration, treatment types, and outcome measures were utilized to analyze the study. Two authors carried out the process of quality assessment, literature selection, as well as data extraction. Any arising difference was resolved by discussion with the help of a third reviewer.

\section{Data synthesis and analyses}

We conducted the statistical analyses on the basis of the Review Manager Software (RevMan5.3) offered by the Cochrane Collaboration. Binary classification data were presented as the risk differences (RD) and its 95\% CI. For continuous outcomes, we assessed the mean difference (MD) as well as its 95\% CI for the result of meta-analysis. Additionally, we calculated the standardized MD (SMD) in case of the need of different scales.

Chi-squared and $\mathrm{I}^{2}$ tests were used to assess the heterogeneity. We selected consistency model to fit the estimated effects. When there was no significant heterogeneity $\left(p>0.1, \mathrm{I}^{2} \leq 50 \%\right)$, we assumed the fixed-effects model would be met. And if statistical heterogeneity was identified $\left(p \leq 0.1, \quad \mathrm{I}^{2}>50 \%\right)$, we detected subgroup analyses to find the sources of heterogeneity on the basis of interventions. In addition, we used combined random-effects model due to fail to identify the sources.

\section{Results}

Search results

The abovementioned search identified 563 articles. After screening duplicates, 506 studies were kept and then 475 articles were excluded due to irrelevant citation, leaving 
31 articles for further evaluation. During full-text screening, 14 articles were excluded. Finally, we included 14 RCTs published from 1999 and 2017 in the review (Fig. 1).

\section{Quality assessment}

The overall results of quality evaluation were presented in Fig. 2 from included studies. Single-blind trials and double-blind trails were applied in 2 trials $[13,14]$ and 4 trials [15-18], respectively. 7 trials [13-16, 18-20] in terms of randomization were used with random number tables, computer-generated random sequence, and block randomization, while no description about the process of randomization was mentioned from other studies. Patients were distributed to 3 trials $[15,18,20]$ by sealed envelope and 1 trial [13] by secure website. Comparable baselines exist in all trials. And analysis of blinded data was independent from all trials of studies. No incomplete report nor selective report were observed. Generally, the studies with moderate quality were included in this meta-analysis.

\section{Study characteristics}

Table 1 summarized the characteristics of included studies. In total, 1864 patients (randomized population, 913 and 951 respectively in Nicorandil and control group) receiving PCI were involved in the current analysis. Patients had an average age that ranged from 57.6 to 71 years, with follow-up duration varied from 1 month to 5 years and a sample size that ranged from 20 to 408. Characteristics of experimental interventions as well as control interventions were

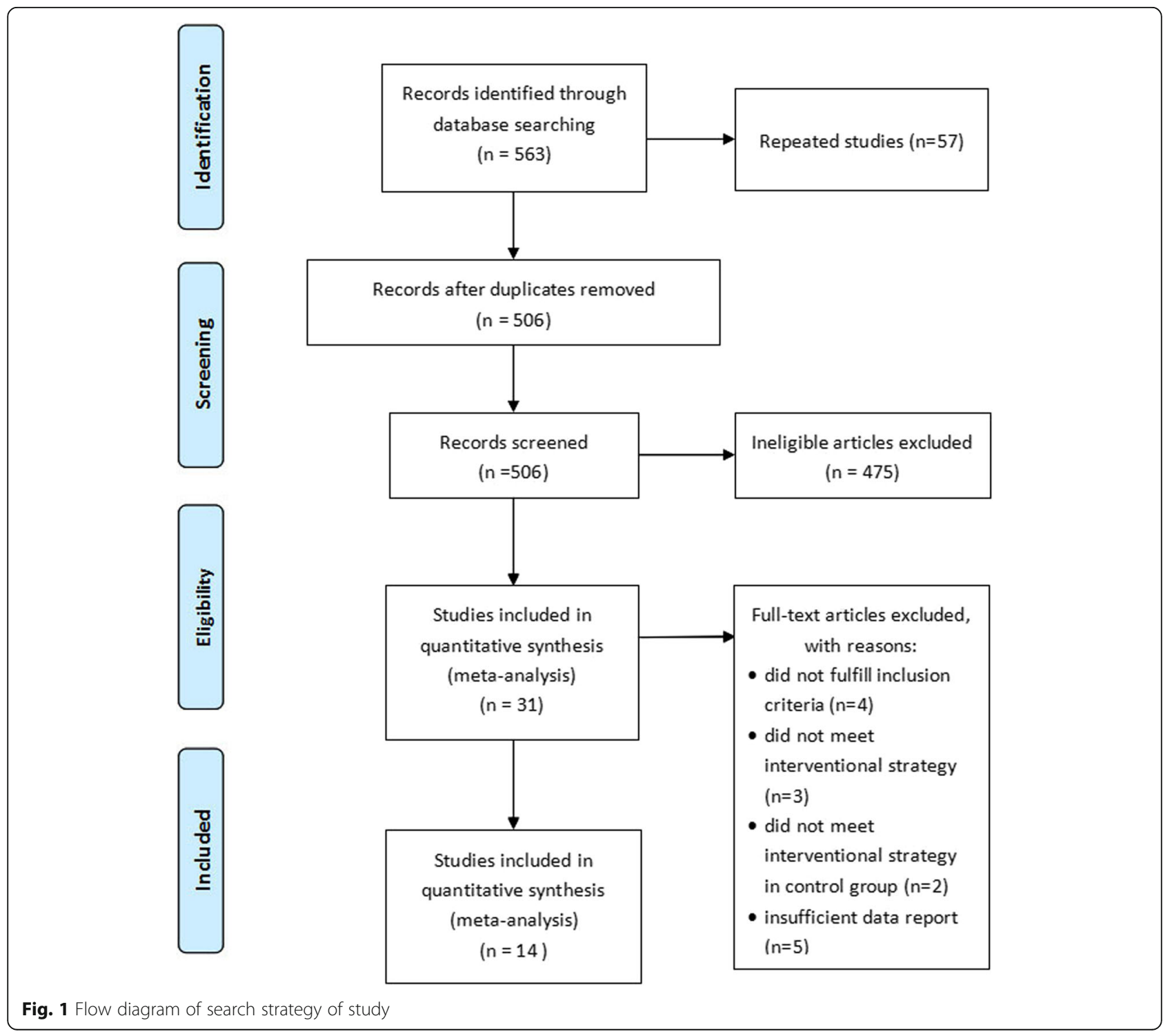




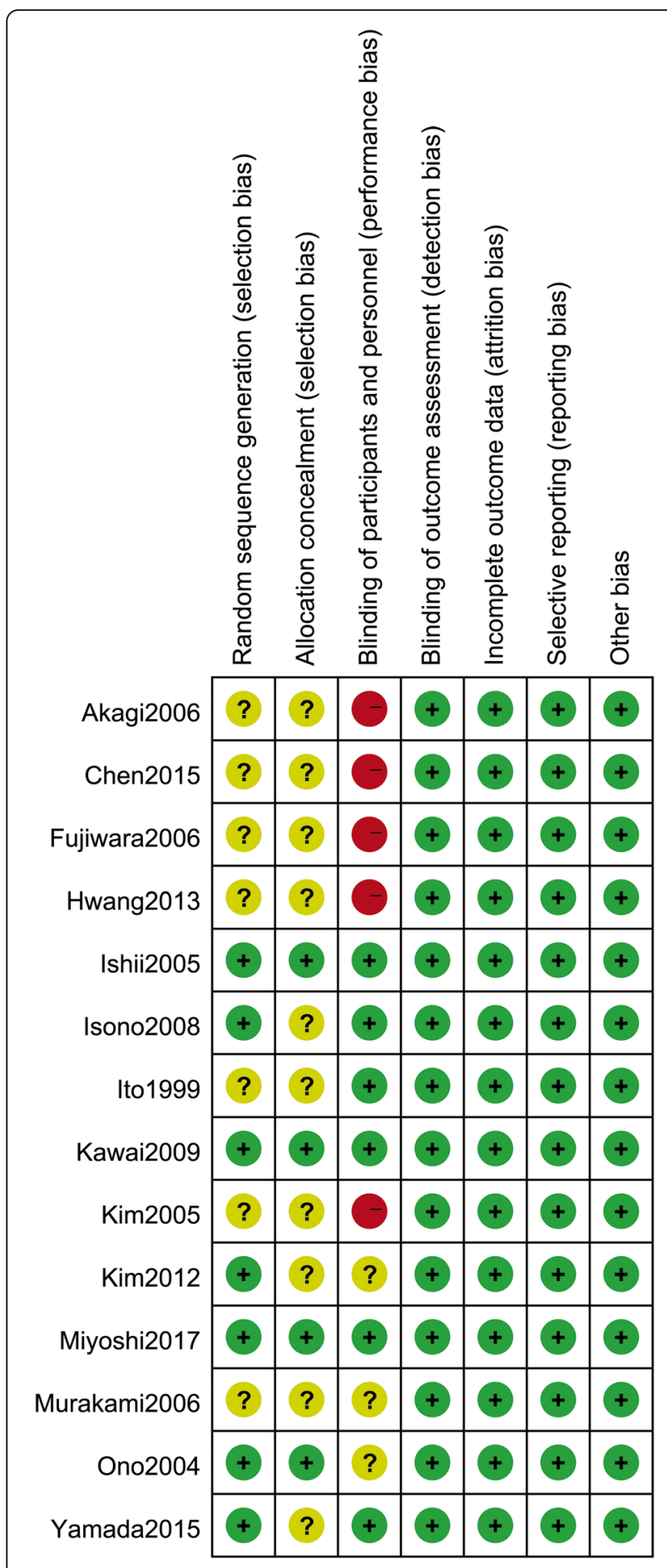

Fig. 2 Methodological quality assessment for each included study

different across the studies. Additionally, several differences were found or observed among the studies with regard of Nicorandil interventions: the routes of administration of Nicorandil, such as intravenous, intracoronary and oral routes; the administration of Nicorandil before or after PCI; the dosage of Nicorandil varied. Most of the included studies used intravenous drip infusion $(4 \mathrm{mg} /$ hour) before PCI for $48 \mathrm{~h}, 2 \mathrm{mg}$ intracoronary and oral $15 \mathrm{mg} /$ day after PCI as intervention program.

\section{Main findings and synthesis of results LVEF}

Eight RCTs [14, 16, 17, 20-24] reported 243 and 247 patients respectively who received PCI in Nicorandil and control group. Statistical heterogeneity was observed among meta-analysis showed between the two studies $\left(P<0.00001, \mathrm{I}^{2}=86 \%\right)$, with the use of random effect model for merging, showing there was significant statistical difference of LVEF when comparing the two groups $(\mathrm{MD}=2.67,95 \% \mathrm{CI}(0.41,4.92), P=0.02)$, as shown in Fig. 3

\section{Peak CK-MB value and peak CTnl value}

The peak CK-MB value was evaluated in 470 patients from 5 RCTs $[16,22,24-26]$. The peak cTnI value was evaluated in 374 patients from 4 RCTs $[16,22,25,26]$. The result showed there was significant statistical difference of peak CK-MB value between Nicorandil group and control group (SMD $=-0.29$, 95\% CI $(-0.47,-0.10), P=0.002)$. Nevertheless, no significant statistical difference was found in terms of peak cTnI value $(\mathrm{SMD}=-0.18,95 \% \mathrm{CI}(-0.39,0.02), P=0.08)$, as shown in Fig. 4.

\section{Major adverse cardiovascular events}

9 RCTs [13-15, 17-20, 22, 24, 26] revealed 785 and 797 patients respectively who received PCI in Nicorandil and control group, with 94 and 135 patients with major adverse cardiovascular events. Statistical heterogeneity was identified among the present meta-analysis comparing the studies $\left(P=0.01, \mathrm{I}^{2}=59 \%\right)$, with the application of random effect model for merging, showing there was significant statistical difference with regard to MACEs rate when comparing two groups $(\mathrm{RD}=-0.04,95 \% \mathrm{CI}$ $(-0.08,-0.00), P=0.04)$, as shown in Fig. 5.

\section{Discussion}

PCI refers to the catheter through a variety of ways to expand the narrow coronary artery, with an attempt to achieve the lifting of the narrow, improve the treatment of myocardial blood supply. As a valid and alternative approach for patients harboring coronary artery anomalies, it can significantly reduce the mortality rate. However, CK-MB, cTnI and other manifestations of myocardial injury is inevitable, leading to poor prognosis. The aggregated results from the present meta-analysis showed that the level of CK-MB in patients from Nicorandil group were associated with lower trend as compared with those in the control group. In 


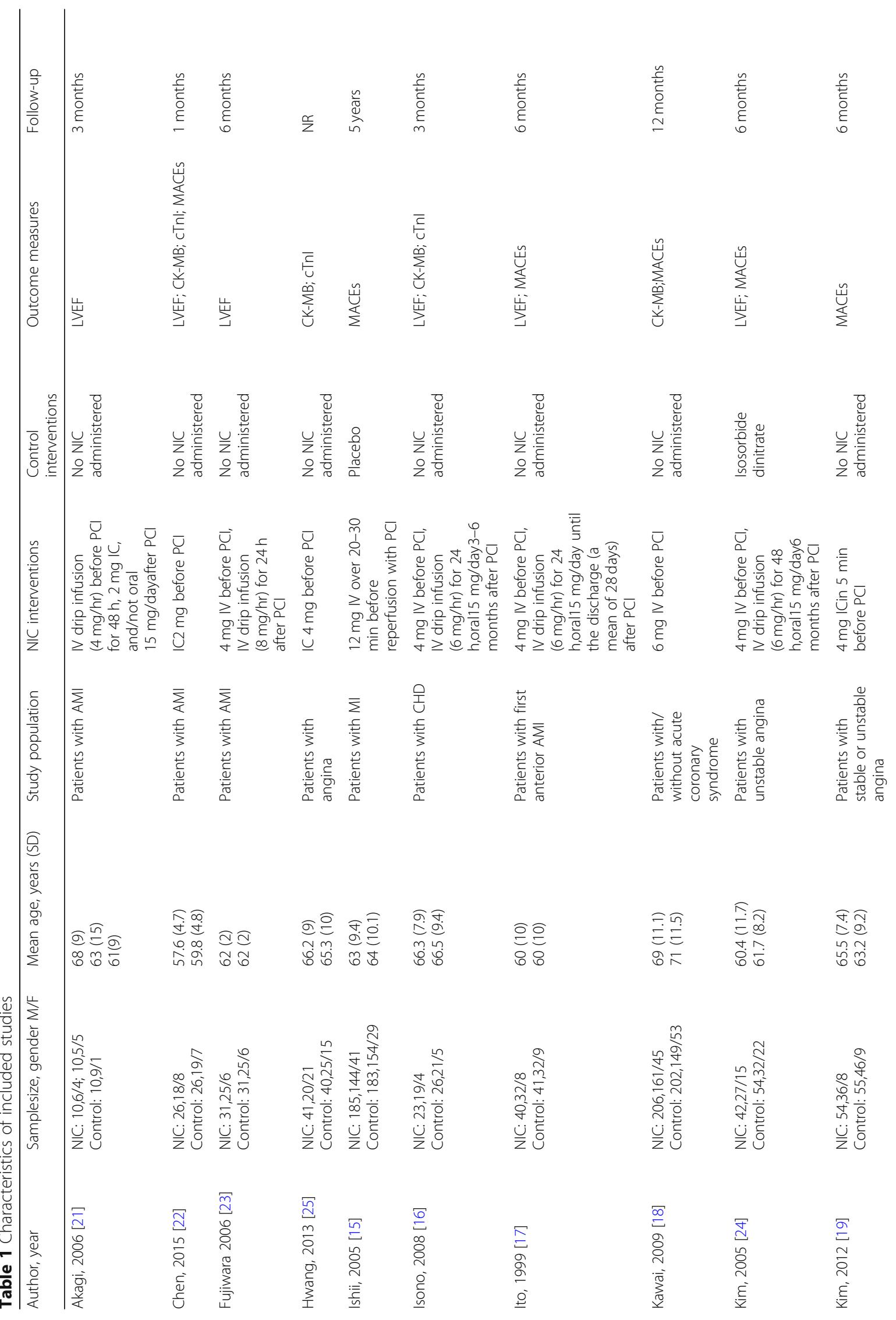


Zhao et al. BMC Cardiovascular Disorders (2019) 19:144

Page 6 of 10

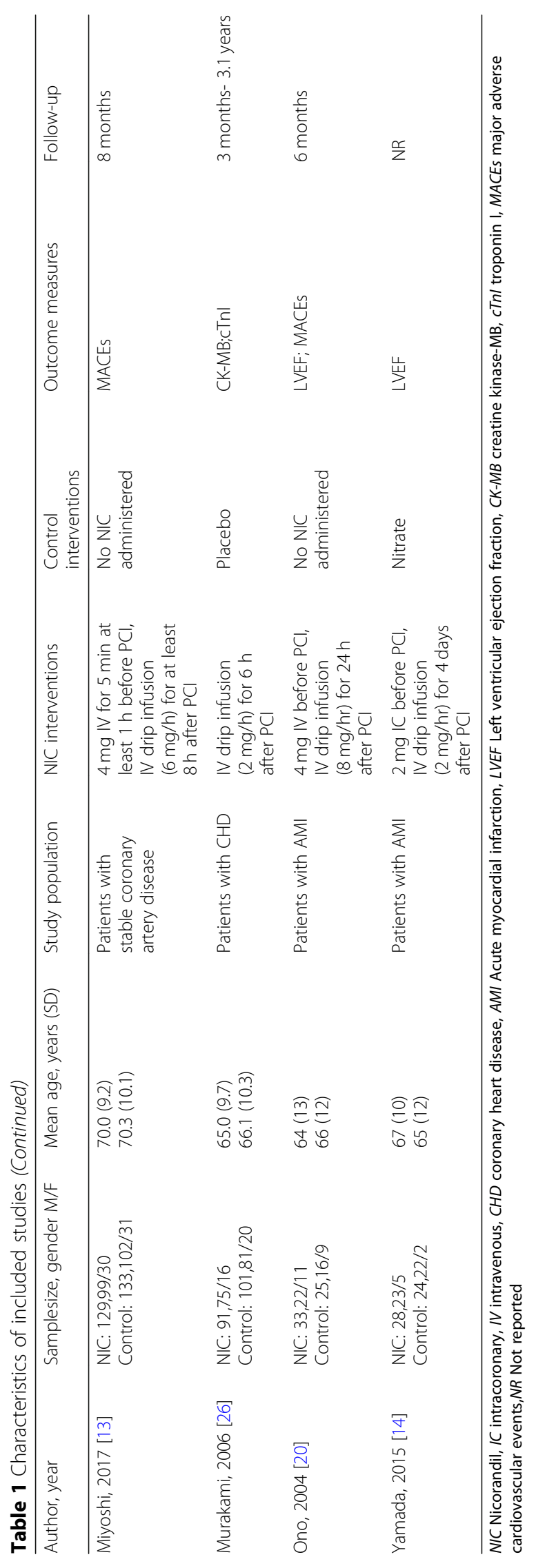




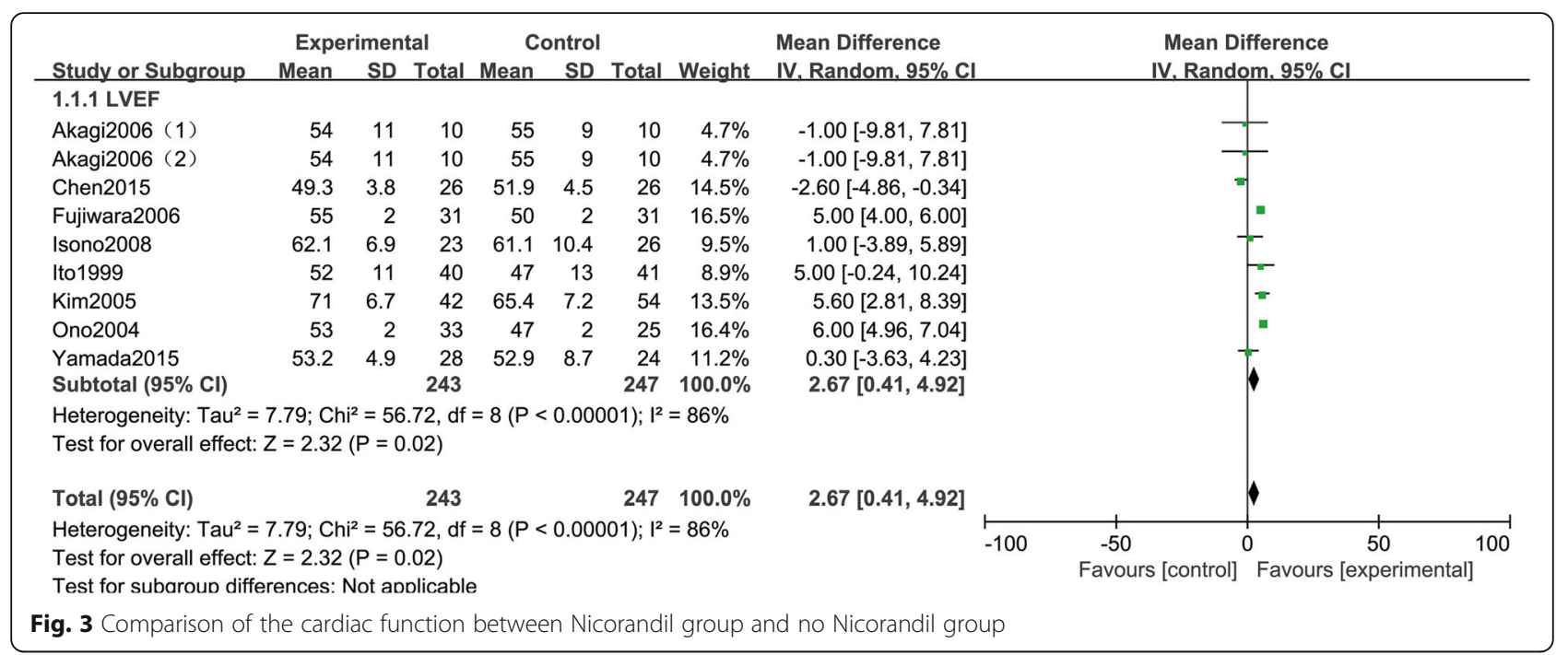

addition, 8 RCTs included in the meta-analysis showed that the left ventricular ejection function of the patients in Nicorandil group was significantly better than that in control group, and also demonstrated that the patients in Nicorandil group had stronger myocardial contractility. It is pivotal to note that the long-term prognosis such as patient mortality, re-hospitalization rate and so on were significantly lower in patients given Nicorandil than in controls. Our meta-analysis failed to show Nicorandil associated with statistically reduced cTnI, but Yang [27] reported that large doses of Nicorandil were associated with a lower incidence of serum cTnI than normal upper limit 3 times compared with low-dose Nicorandil and control groups, and the protective effect of Nicorandil on the myocardial injury was improved to some extent. Therefore, more well designed studies are needed to confirm the effect of Nicorandil in the setting of PCI.

According to several previous studies [28-30], apart from its cardioprotective effect, Nicorandil improves microvascular dysfunction. Moreover, it can be administered intravenously as an intracoronary infusion or before starting PCI. Confirmation has been gained in terms of the effectiveness of Nicorandil on stable angina in a large clinical randomized controlled trial of IONA [31] wherein there included a positive exercise test already on the optimal antianginal drug therapy or with patients harboring established coronary heart disease (previous MI, CABG). Patients in Nicorandil group $(N=2565)$ versus placebo $(N=2561)$ was significantly

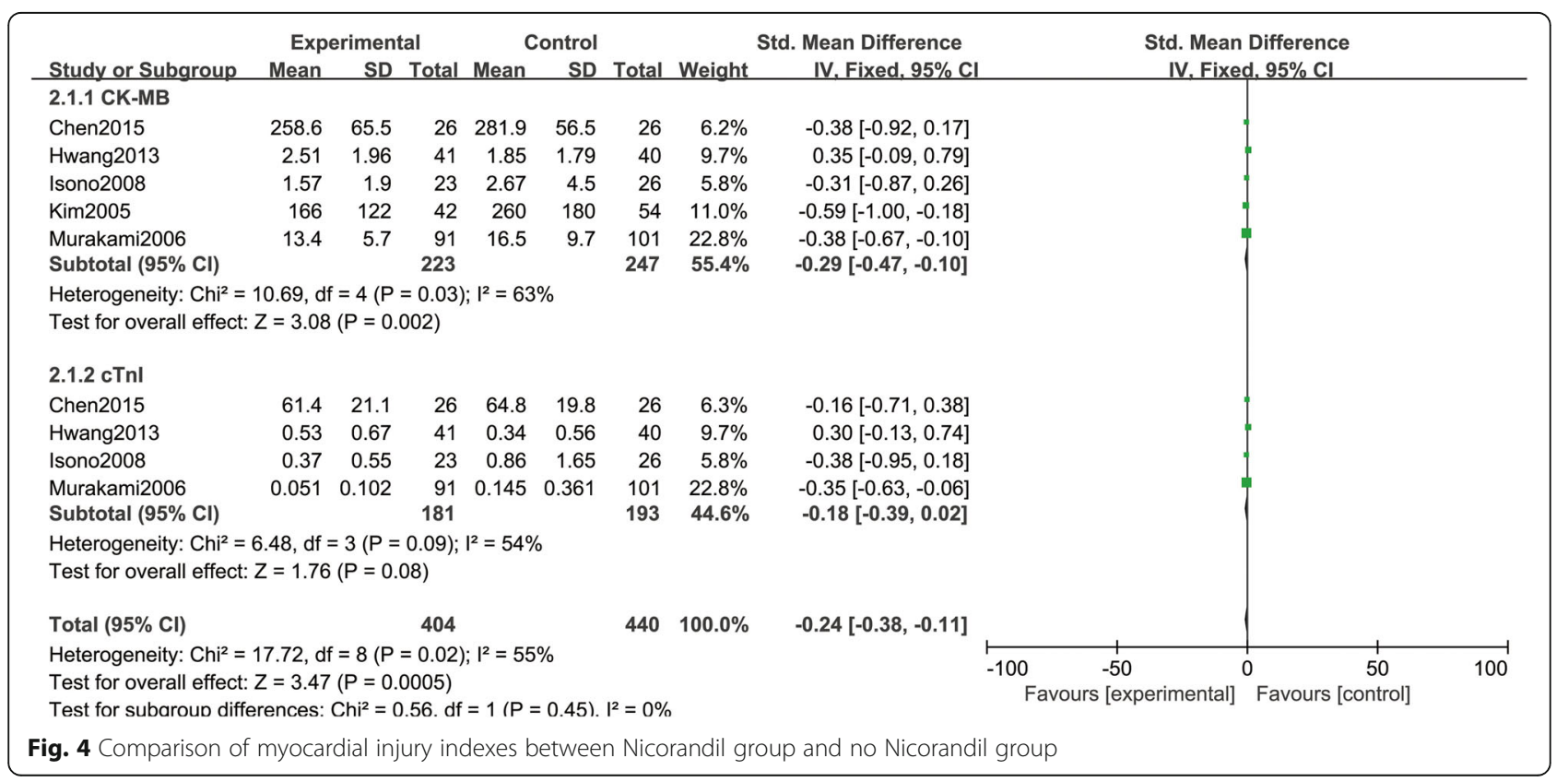




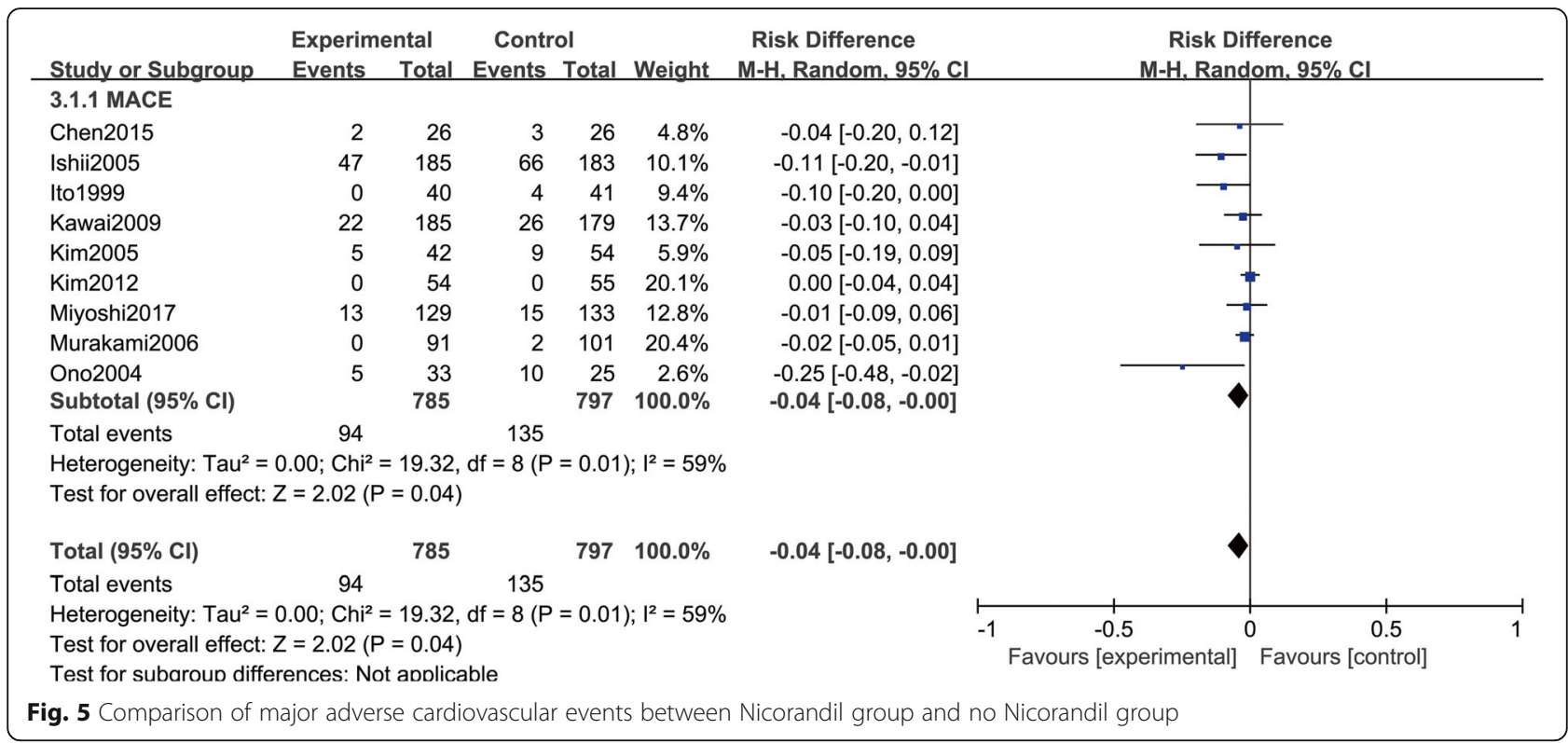

reduced in the incidence of adverse cardiac events, but there was no significant difference with regard to the mortality between the two groups $(4.3 \%$ Vs. $5.0 \%$, $\mathrm{HR}=0.85, P=0.222)$, which may be related to short follow-up time. It was consistent with subgroup analyses in a Japanese retrospective study (JCAD) [32], the study reported that Nicorandil in treating patients with coronary artery disease can significantly reduce cardiac death, fatal myocardial infarction, cerebrovascular death, congestive heart failure, re-admission rate and other end-point indicators.

The mechanisms of Nicorandil in reduced myocardium injury as well as improved heart function are suggested to attribute to several factors. First, Nicorandil pharmacologically dilates the coronary artery microvessels with a diameter of $<100 \mu \mathrm{m}$, increases coronary blood flow and decreases the heart preload [33]. Second, Nicorandil has myocardial protection in acute ischemic preconditioning, which exert effect of prevention of reperfusion injury as well as protection of myocardium from ischemic injury via ATP-sensitive $\mathrm{K}+$ channels. In ischemic myocardium, intracellular ATP depletion can activate the K-ATP channels, leading to increased outflow of potassium, thereby the duration of the action potential shortens and the amount of calcium that flows into the myocytes reduces. Inhibiting and reducing the role of calcium in cardiomyocytes is thought to be a mechanism of cardioprotective effects on ischemic heart, and Nicorandil contributes to this effect as K-ATP channel opener. Since microvascular obstruction after myocardial infarction was associated with left ventricular remodelling, Nicorandil could improve left ventricular function for inhibiting the progression of microvascular damage and reperfusion arrhythmia. A previous study reported that intravenous infusion of Nicorandil in acute MI reduced myocardial injury and improved cardiac function, as shown by echocardiographic regional wall motion scoring and quantitative thallium single-photon emission computerized tomography analysis, respectively [34]. However, several studies [14, 21, 22] showed that Nicorandil did not significantly improve LVEF, which may be related to shorter follow-up time and baseline differences in the study population. In conclusion, LVEF is one of the most important factors affecting the prognosis of patients harboring coronary artery disease, and its improvement may lead to a decrease in the incidence of adverse cardiac events.

In addition, oral administration of Nicorandil can positively affect the activity of the cardiac sympathetic nerve [35], long-term administration of Nicorandil has good compliance with less adverse reactions [36]. Nicorandil has antihypertensive effects. These are also the reasons why Nicorandil could improve the prognosis of patients who are diagnosed with coronary artery disease.

There were several limitations in current analysis that should be acknowledged. First, there were only few eligible trials in some subgroups of meta-analyses, given the small and single-center nature of the current 14 RCTs with various quality, and only 2 RCTs $[13,18]$ were multicenter, large-scale clinical trials. Only 3 studies took the allocation of double-blind and hidden, the rest of the allocation of blind or hidden cases were unclear; only 7 studies of random grouping method were correct, with the rest insufficient. Second, there was a baseline inconsistency in the study, i.e. inconsistent intervention measures, including coronary and intravenous administration, continuous intravenous 
administration, dose of Nicorandil and subsequent long-term oral administration. Based on the aggregated results of current study, Nicorandil efficacy and dose-dependent relationship were not significant, with only part of the collection of fixed doses of Nicorandil research, thereby the current dose-dependent analysis of the relationship was inconclusive. Relevant researches were needed for further improvements in terms of the following aspects: right random allocation and allocation of hidden programs, larger sample size, longer follow-ups to further observe the short-term as well as long-term effects, identify the role of different routes of administration and the efficacy on dose, more thorough and comprehensive assessment with regard to the efficacy as well as the adverse reactions of Nicorandil.

\section{Conclusions}

Moderate evidence has been collected in this systematic review that intracoronary/intravenous injection or oral Nicorandil is currently an acceptable and effective adjunctive therapy in patients who suffer from coronary artery disease and undergo elective PCI. It appears to be associated with the suppression of myocardium injury, the improved left ventricular function, and reduced incidence of adverse cardiac events at long term. However, considering the poor quality of the papers evaluated, additional high-quality RCTs are in great demand to confirm the effect of Nicorandil therapy accompanied with PCI in treating patients who suffer from coronary artery disease.

\section{Acknowledgements \\ None.}

\section{Funding}

This study was supported by National Natural Science Foundation of China (81670214), the Beijing Natural Science Foundation (7172080), and the 1315 personnel training plan (CYMY-2017-03).

\section{Availability of data and materials}

Data sharing is not applicable to this article as no datasets were generated or analyzed during the current study.

\section{Authors' contributions}

X-T Z and C-F Z have made substantial contributions to conception and design of the study; C-F Z and Q-J L searched literature, extracted data from the collected literature and analyzed the data; $X-T Z Z$ wrote the manuscript; C-F $Z$ revised the manuscript; All authors approved the final version of the manuscript.

\section{Ethics approval and consent to participate}

Not applicable.

\section{Consent for publication}

Not applicable.

\section{Competing interests}

The authors declare that they have no competing interests.

\section{Publisher's Note}

Springer Nature remains neutral with regard to jurisdictional claims in published maps and institutional affiliations.

\section{Author details}

${ }^{1}$ Department of Cardiology, Beijing Chao-Yang Hospital, Captial Medical University, 8 Gongti Nanlu Road, Chaoyang District, Beijing 100020, NO, China. ${ }^{2}$ Department of Internal Medicine, General Political Department Hospital of Chinese PLA, Beijing, China. ${ }^{3}$ National Institute for Radiological Protection, Chinese Center for Disease Control and Prevention, Chinese Center for Medical Response to Radiation Emergency, No. 2 Xinkang Street, Deshengmenwai, Beijing 100088, China.

Received: 8 November 2017 Accepted: 9 April 2019

Published online: 14 June 2019

\section{References}

1. Wong WP, Feng J, Pwee KH, Lim J. A systematic review of economic evaluations of cardiac rehabilitation. BMC Health Serv Res. 2012;12:1-8.

2. Grüntzig AR, Senning A, Siegenthaler WE. Nonoperative dilatation of coronary-artery stenosis: percutaneous transluminal coronary angioplasty. N Engl J Med. 1979;301:61.

3. Faxon DP. Coronary interventions and their impact on post myocardial infarction survival. Clin Cardiol. 2005:28(11 Suppl 1):138-44.

4. Gillies MA, Shah AS, Mullenheim J, Tricklebank S, Owen T, Antonelli J, Strachan F, Mills NL, Pearse RM. Perioperative myocardial injury in patients receiving cardiac output-guided haemodynamic therapy: a substudy of the OPTIMISE trial. Br J Anaesth. 2015;115:227-33.

5. Verma S, Pw F, Rd W, J B, Maitland A, Li R-K, Dhillon B, Yau TM. Fundamentals of reperfusion injury for the clinical cardiologist. Circulation. 2002;105:2332-6.

6. Zeymer U, Suryapranata H, Jp OG, Davies J, Rasmanis G, Linssen G, Tebbe U Schroder R, Tiemann R, Machnig T. The $\mathrm{Na}(+) / \mathrm{H}(+)$ exchange inhibitor eniporide as an adjunct to early reperfusion therapy for acute myocardial infarction. Results of the evaluation of the safety and cardioprotective effects of eniporide in acute myocardial infarction (ESCAMI) trial. J Am Coll Cardiol. 2001:38:1644.

7. European Study of Prevention of Infarct with Molsidomine (ESPRIM) Group. The ESPRIM trial: short-term treatment of acute myocardial infarction with molsidomine. The Lancet. 1994;344(8915):91-97

8. Wall TC, Rm C, J B, Jd T, M T, M S, G G, Md C, M S, Leimberger JD. Intravenous Fluosol in the treatment of acute myocardial infarction. Results of the thrombolysis and angioplasty in myocardial infarction 9 trial. TAMI 9 research group. Circulation. 1994;90:114-20.

9. Matsuo H, Watanabe S, Segawa T, Yasuda S, Hirose T, Iwama M, Tanaka S, Yamaki T, Matsuno Y, Tomita M. Evidence of pharmacologic preconditioning during PTCA by intravenous pretreatment with ATPsensitive K+ channel opener nicorandil. Eur Heart J. 2003;24:1296-303.

10. Ishida H, Higashijima N, Hirota Y, Genka C, Nakazawa H, Nakaya H, Sato T. Nicorandil attenuates the mitochondrial $\mathrm{Ca} 2+$ overload with accompanying depolarization of the mitochondrial membrane in the heart. Naunyn Schmiedeberg's Arch Pharmacol. 2004;369:192-7.

11. Sugimoto KIH, Iwakura K. Intravenous Nicorandil in conjunction with coronary reperfusion therapy is associated with better clinical and functional outcomes in patients with acute myocardial infarction. Circ J. 2003;67(4):295-300.

12. Mizumura T, Nithipatikom K, Gross GJ. Infarct size-reducing effect of nicorandil is mediated by the KATP channel but not by its nitrate-like properties in dogs. Cardiovasc Res. 1996;32:274-85.

13. Miyoshi T, Ejiri K, Kohno K, Nakahama M, Doi M, Munemasa M, Murakami M, Takaishi A, Kawai Y, Sato T. Effect of remote ischemia or nicorandil on myocardial injury following percutaneous coronary intervention in patients with stable coronary artery disease: A randomized controlled trial. Int J Cardiol. 2017;236:36-42.

14. Yamada K, Isobe S, Ishii H, Yokouchi K, Iwata H, Sawada K, Murohara T. Impacts of nicorandil on infarct myocardium in comparison with nitrate: assessed by cardiac magnetic resonance imaging. Heart Vessel. 2016;31:1430-7

15. Ishii H, Ichimiya S, Kanashiro M, Amano T, Imai K, Murohara T, Matsubara T. Impact of a single intravenous administration of nicorandil before 
reperfusion in patients with ST-segment-elevation myocardial infarction. Circulation. 2005;112:1284.

16. Isono T, Kamihata H, Sutani Y, Motohiro M, Yamamoto S, Kyoui S, Iharada $Y$, Kurimoto K, Hara K, Takahashi H. Nicorandil suppressed myocardial injury after percutaneous coronary intervention. Int J Cardiol. 2008;123:123-8.

17. Ito H, Taniyama Y, Iwakura K, Nishikawa N, Masuyama T, Kuzuya T, Hori M, Higashino Y, Fujii K, Minamino T. Intravenous nicorandil can preserve microvascular integrity and myocardial viability in patients with reperfused anterior wall myocardial infarction - its dependency on vessel size and the involvement of the ATP-sensitive potassium channels. J Am Coll Cardiol. 1999;33:654-60.

18. Kawai Y, Hisamatsu K, Matsubara H, Dan K, Akagi S, Miyaji K, Munemasa M, Fujimoto Y, Kusano KF, Ohe T. Intravenous administration of nicorandil immediately before percutaneous coronary intervention can prevent slow coronary flow phenomenon. Eur Heart J. 2009:30:765-72.

19. Kim SJ, Kim W, Woo JS, Ha SJ, Kang WY, Hwang SH, Kang DG, Lee SU, Cho SK, Im JS. Effect of myocardial protection of intracoronary adenosine and nicorandil injection in patients undergoing non-urgent percutaneous coronary intervention: a randomized controlled trial. Int J Cardiol. 2012;158:88.

20. Ono H, Osanai T, Ishizaka H, Hanada H, Kamada T, Onodera H, Fujita N, Sasaki S, Matsunaga T, Okumura K. Nicorandil improves cardiac function and clinical outcome in patients with acute myocardial infarction undergoing primary percutaneous coronary intervention: role of inhibitory effect on reactive oxygen species formation. Am Heart J. 2004;148:611.

21. Akagi T, Sarazawa K, Inai Y, Kitagawa M, Takahashi N, Hamanaka I, Yamazaki T, Takebe M, Hama N, Hiraoka Y. Continuous administration of nicorandil decreases QT dispersion during the chronic phase of acute myocardial infarction. Int Heart J. 2006:47:351.

22. Chen C, Xianghua FU, Wei LI, Jia X, Bai S, Geng W, Xing K. Intracoronary administration of anisodamine and nicorandil in individuals undergoing primary percutaneous coronary intervention for acute inferior myocardial infarction: A randomized factorial trial. Exp Ther Med. 2015;10:1059.

23. Fujiwara T, Matsunaga T, Kameda K, Abe N, Ono H, Higuma T, Yokoyama J, Hanada H, Osanai T, Okumura K. Nicorandil suppresses the increases in plasma level of matrix metalloproteinase activity and attenuates left ventricular remodeling in patients with acute myocardial infarction. Heart Vessel. 2007;22:303-9.

24. Kim JH, Jeong MHYun KH, Kim KH, Kang DK, Hong SN, Lim SY, Lee SH, Lee YS, Hong YJ, Park HW. Myocardial protective effects of nicorandil during percutaneous coronary intervention in patients with unstable angina. Circ J. 2005;69:306-10

25. Hwang J, Han CL, Kim BW, Mi JY, Park JS, Park JH, Lee HW, Oh J, Choi JH, Cha KS. The effect on periprocedural myocardial infarction of intra-coronary nicorandil prior to percutaneous coronary intervention in stable and unstable angina. J Cardiol. 2013;62:77-81.

26. Murakami M, Iwasaki K, Kusachi S, Hina K, Hirota M, Hirohata S, Kamikawa S, Sangawa M, Yamamoto K, Shiratori Y. Nicorandil reduces the incidence of minor cardiac marker elevation after coronary stenting. Int J Cardiol. 2006;107:48-53.

27. Yang J, Zhang J, Cui W, Liu F, Xie R, Yang X, Gu G, Zheng H, Lu J, Yang X. Cardioprotective effects of single oral dose of nicorandil before selective percutaneous coronary intervention. Anatol J Cardiol. 2015;15:125-31.

28. lida S, Kinoshita H, Holford NHG. Population pharmacokinetic and pharmacodynamic modelling of the effects of nicorandil in the treatment of acute heart failure. Br J Clin Pharmacol. 2008;66:352.

29. Imakura K, Ito H, Okamura A, Koyama Y, Date M, Higuchi Y, Inoue K, Kimura $\mathrm{R}$, Nagai $\mathrm{H}$, Imai M. Nicorandil treatment in patients with acute myocardial infarction: a meta-analysis. Circ J. 2009;73:925-31.

30. Ishii H, Ichimiya S, Kanashiro M, Amano T, Matsubara T, Murohara T. Effects of intravenous nicorandil before reperfusion for acute myocardial infarction in patients with stress hyperglycemia. Diabetes Care. 2006;29:202-6.

31. The IONA Study Group. Effect of nicorandil on coronary events in patients with stable angina: the impact of Nicorandil in angina (IONA) randomised trial. Lancet. 2002;359:1269-75.

32. Horinaka S, A Y, Yagi H, Ishimitsu T, Yamazaki T, Suzuki S, Kohro T, Nagai R. Effects of nicorandil on cardiovascular events in patients with coronary artery disease in the Japanese coronary artery disease (JCAD) study. Circ J. 2010;74(3):503-9.

33. Akai K, Wang Y, Sato K, Sekiguchi N, Sugimura A, Kumagai T, Komaru T, Kanatsuka $\mathrm{H}$, Shirato K. Vasodilatory effect of nicorandil on coronary arterial microvessels: its dependency on vessel size and the involvement of the ATP-sensitive potassium channels. J Cardiovasc Pharmacol. 1995:26:541-7.

34. Wang A, Chen F, Xie Y, Guo Z, Yu Y. Protective mechanism of nicorandil on rat myocardial ischemia-reperfusion. J Cardiovasc Med. 2012;13:511.

35. Kasama S, Toyama T, Sumino H, Kumakura H, Takayama Y, Ichikawa S, Suzuki T, Kurabayashi M. Long-term nicorandil therapy improves cardiac sympathetic nerve activity after reperfusion therapy in patients with first acute myocardial infarction. J Nucl Med. 2007:48:1676.

36. Krumenacker $\mathrm{M}$, Roland E. Clinical profile of nicorandil: an overview of its hemodynamic properties and therapeutic efficacy. J Cardiovasc Pharmacol. 1992;20(Suppl 3):S93.

\section{Ready to submit your research? Choose BMC and benefit from:}

- fast, convenient online submission

- thorough peer review by experienced researchers in your field

- rapid publication on acceptance

- support for research data, including large and complex data types

- gold Open Access which fosters wider collaboration and increased citations

- maximum visibility for your research: over $100 \mathrm{M}$ website views per year

At BMC, research is always in progress.

Learn more biomedcentral.com/submissions 